\title{
Research on Automotive Interior Optimization Based on Human Factors Engineering
}

\author{
Sun Wenlong \\ (NanChang Institute of Science \& Technology, Nanchang,330108; Jiangxi Engineering Laboratory \\ of Digital Manufacturing for Automobile Parts)
}

Keywords: Human factors engineering; Automotive interior; Color; Material; Personality

\begin{abstract}
The automotive interior brings people an intuitive and comfortable feeling that directly affects the image of the automobile. With the increase of consumer spending power, consumers are paying more and more attention to the appearance of the automobile interior while attaching attention to the performance of the automobile. In the pursuit of comfort, car interior decorators have begun to focus on the individual needs of consumers. Therefore, the concept of car sales for car dealers can not merely stay on the choice of past sales strategies, but also need to be more meticulous and personalized, which can gain the favor of consumers before they can stand out among many competitors. This article starting from the human factors project, and the perspective of automotive interior design, puts forward opinions and viewpoints on the personalized design of automotive interiors according to colors and materials, hoping to provide some feasible suggestions for the personalized design of automobile interiors in China.
\end{abstract}

\section{Introduction}

The ability of consumers to buy cars is becoming more and more powerful. While buying cars, they also love to show their individuality and differentness. To prevent their cars from being similar to others, more and more people are pursuing their car interiors. Personalized design are increasingly demanding, in the same price situation, if a brand of car interior personalized design does not meet consumer expectations, then consumers are likely to turn to others, therefore, companies should pay attention to the personalized design of car interiors. Only after fully paying attention to the personalized needs of consumers can they better meet the needs of consumers and be able to accurately grasp their Preference and buying preferences can be invincible in the fierce market competition. Therefore, this paperanalyzes the personal customization of peoplebased on human factors engineering.

\section{Human Factors Project Introduction}

Human factors engineering is based on human factors and uses human science knowledge such as psychology, physiology, anatomy, anthropometry, etc. to design engineering and operations management, especially safety design and safety management. People-oriented, focusing on improving human performance, preventing human error, and considering the optimization of the overall performance of the human-machine-environment system under the conditions of making the system as safe and comfortable as possible.

It is precisely because of the emphasis on human factors in engineering design and operation management that is the characteristics of the discipline. Therefore, we use human factors engineering as the name of this discipline to highlight the application of human factors in engineering. From a textual point of view, "Human Factors Engineering", as the name suggests, is very easy to think about people's factors, and easily understood and accepted.

"Uniformly considering the optimization of the overall performance of the man-machine-environment system" means that the design of the machine must be consistent with the physiological and psychological characteristics of the person and be conducive to "safe, efficient, and comfortable", that is, "ameneutical", as well as Consider adapting people to machines through 
training and management, ie "personal opportunities". One-sided emphasis on one aspect does not meet human factors engineering principles.

The use of human factors engineering focuses on the interrelationships between humans and the products, equipment, facilities, procedures and environment used in work and daily life. Correct use of human factors engineering will improve work efficiency and quality, and meet people's value needs.

\section{Influence of Automotive Interior Color on Personalized Decoration}

One of the key factors in the design of automotive interiors is the color. The color of the car interior can not be underestimated. The color of the car interiors that were more popular in previous years was dominated by single grayish tones such as black and white. Occasionally, some of the brighter colors were used, but today's interior color selection is no longer limited to the past. A single color tone, but focusing on the use of a variety of colors, automotive interior designers have also found the important role of color in the interior configuration, belonging to the spiritual consumption of car buyers, they also began to realize that from the color point of view Better car interior design with people oriented.

In general, the visual sense of the public is different, so the love for color will also be different, and then there will be great differences in the color choice of car interiors. This difference is generally from the age, gender, occupation Such as different.

People of different ages are divided into four levels: youngsters, young people, middle-aged people and senior citizens. We choose to extract color schemes from their living environment. Solid color car interior is more suitable for owners with children, this color matching to take care of the hearts of minors, seems relatively single, bright. For adult color selection, the brightness is generally lower, purity is relatively lower, and dark colors are matched. This color is more in line with the aesthetic pursuit of adults. Brightness of car interior color, as shown in Figure 1.

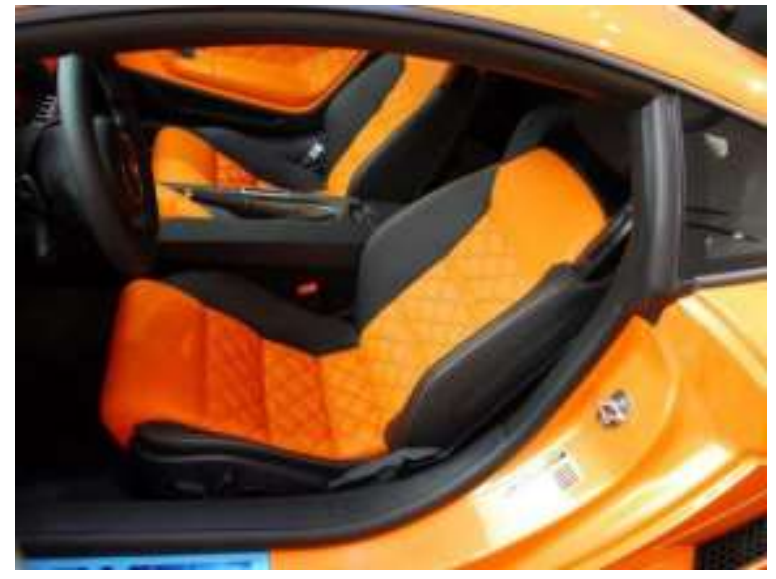

Figure 1 High-brightness car interior color

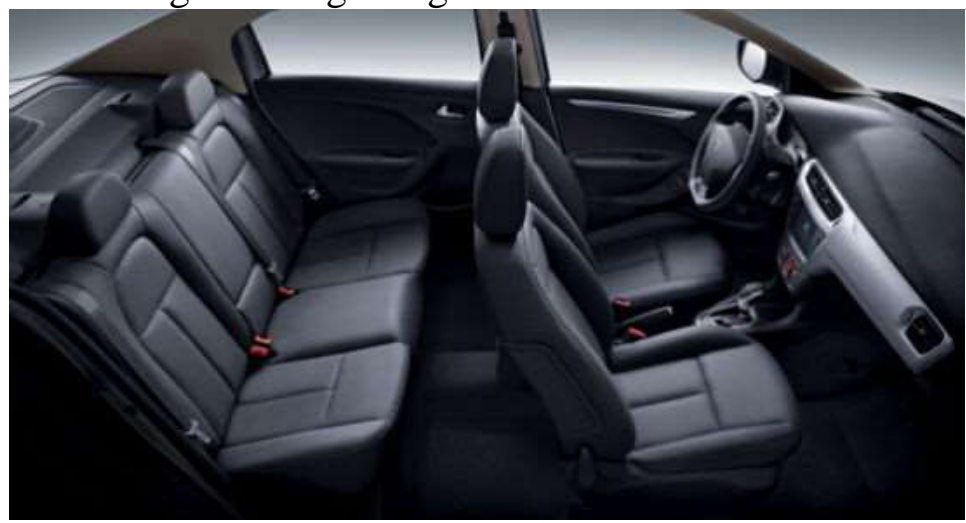

Figure 2 low-light automotive interior color 
The color tone of car interior color design is critical. This car interior color design reflects the color matching of adult sensory pursuits. They often use dark colors as the main color, and the lower purity of gray is also within their range of choice. In general, as people's age increases, the pursuit of color will tend to be primary, and people will use colors that are relatively high purity as key colors to set off their adult maturity and sense of stability.

\section{Influence of Automotive Interior Materials on Personalized Decoration}

Automotive interior design requires the use of a wide variety of materials in the decoration process. According to experience, under normal circumstances, the human mental state will be affected by different automotive interior materials, each material has a synchronous nature. This is as if when we first saw a strange space, we would have different types of feelings. Starting from the overall tone, including the overall setting, and then the guesswork of the type of material, the final decision material. It is also analyzed, such as the shape, texture, color, gloss, etc. of the material.

Take the seat cover as an example. Different materials can bring different style experiences to consumers. For example, cotton seat cover: calm and generous, inexpensive and hard to wear, the seat cover of cotton texture is calm and generous, strong and durable. Not easy to wear. This kind of seat cover is very practical, it can be replaced at any time, and there is no special place to pay attention to in the dismantling process. All kinds of washing methods are applicable, allowing the owner to keep the car clean and fresh.

The seat cover of a car is generally divided into a variety of types such as a cotton seat cover, a blended seat cover, a straw seat cover, and a mixed seat cover leather seat cover. The material of the cotton seat cover is generally made of cotton. The use of this seat cover generally makes the owner calm and generous, and the texture of the seat cover is strong and durable, and has a long service life. There is also a very practical and economical car seat cover that is the seat cover of the blended material. The mixed seat cover can be divided into two types of cotton material plus card and cotton material plus card and polyester blend. Its advantage lies in its very high quality. Strong and easy to clean, but rough texture. The seat cover of this material will not shrink or deform after being washed and can be used by the consumer for a longer period of time. One type of leather seat cover that we have to mention is the leather seat cover. The leather seat cover can be said to be the superstar in all seat cover types. It is not only good-looking but also practical. The most important thing is that it is very high-grade. One of the biggest advantages is that it is cool in winter and cool in summer, and it is suitable for all seasons. It is also very soft and comfortable after you sit down. However, the high price of leather seat covers is not affordable for ordinary people. Only the owners of medium and high-grade cars have the economic strength to use leather seat covers.

\section{Conclusion}

Color is a very intuitive visual experience for human beings, and it is an important factor that directly determines whether car buyers are interested in car products. Therefore, we will talk about whether the matching of colors in car interior design meets the consumer experience and will have a direct impact on the consumer's consumer psychology. And car interior designers need to pay attention to first-hand car users' experience in car interior design. Through understanding the needs of consumers when choosing car interior materials, make different types of choices, along with consumers. The requirements for automotive interiors are also getting higher and higher. In order to further satisfy the consumers, the need for personalized design is elaborated separately from the colors and materials. It is hoped that it can provide some reference for the personalized design of automobile interiors in China.

\section{Acknowledgement}

Project funding: Project at Nanchang institute of science and technology (No.SZZX-17-14) 


\section{References}

[1] Dong S F, Xiao-Qing W U, Feng L F. The reverse design of automotive interior parts Based on reverse engineering[J]. Machinery Design \& Manufacture, 2009.

[2] Liu L, Tang J Y, Gao J. Research on automobile interior design based on operation process of kansei engineering[J]. Modern Manufacturing Engineering, 2010.

[3] Liu L, Tang J Y, Gao J. Research on automobile interior design based on operation process of kansei engineering[J]. Modern Manufacturing Engineering, 2010.

[4] Lin Z Y, Hou W L. Optimization Design for Automotive Interior Trimmings Based on Moldflow[J]. Key Engineering Materials, 2012, 522:515-519.

[5] Tan Y W, Wang Y M, Zhou G, et al. Automotive Interior Parts Molding Process Simulation and Parameter Optimization[J]. Advanced Materials Research, 2013, 734-737(734-737):2725-2729.

[6] Zhu L H, Zhao H, Yin X C, et al. Computer Aided Automotive Interior Design for Kansei Engineering[J]. Applied Mechanics \& Materials, 2011, 130-134:504-507.

[7] Koizumi K, Kanke R. Research on Automobile Exterior Color and Interior Color, Matching[J]. International Journal of Engineering Research \& Applications, 2014, 4(8).

[8] Gao Z, Ang Y E, Jiangnan Y U, et al. On the Design Method of Automotive Interiors Modeling Based on Virtual Human Technology[J]. Journal of Changzhou Institute of Technology, 2014.

[9] Xu H. Mechanical Properties of PMMA Used for Automotive Interior Based on Outside Mold Decoration[J]. Engineering Plastics Application, 2016.

[10] Yao L, Zhang G. Study on Automotive Interior Structure-Borne Sound Based on TPA[M]// Proceedings of the FISITA 2012 World Automotive Congress. Springer Berlin Heidelberg, 2013:457-469.

[11]Luo W, Luo X. User Experience Research on Automotive Interior Lighting Design[C]// International Conference on Applied Human Factors and Ergonomics. Springer, Cham, 2017:240-246.

[12]Luo W, Luo X. User Experience Research on Automotive Interior Lighting Design[J]. 2017. 